\title{
Response of the Pulp and Dentin to Contact with Filling Materials
}

\author{
JAMES K. AVERY \\ University of Michigan, School of Dentistry, Ann Arbor, Michigan 48104, USA
}

I have four objectives for this research report concerning the responses of pulp and dentin to dental materials:

1. to discuss the fine structure of the pulp and dentin to understand better why they respond as they do to various pharmacological agents;

2. to describe a theory of dental repair which occurs as a response to external stimuli;

3. to make a plea for uniform testing procedures such as those suggested by the recommended "Standards practices for clinical evaluation of dental materials and devices" as published in the Journal of the American Dental Association in February 1972; and

4. to discuss the pulpal and dentin response to the more common restorative materials of today such as polycarboxylates, composites, silicates, calcium hydroxide compounds, and zinc oxide eugenol.

\section{Study of Ultrastructure of the Dentin and Pulp}

In consideration of the first point of our objectives, we are aware that the pulp organ throughout life is composed of cells, intercellular substance, and fibers. We know that the cells of the pulp organ are fibrocytes or fibroblasts located throughout the pulp and that the specialized odontoblasts are located along the periphery of the pulp at the border of the predentin. It is probable that all of these cells have a common origin. The fibroblasts of the pulp produce collagen that may appear in the pulp as diffuse fibers, or it may form bundles throughout the pulp.

Professor Baume, who was originally scheduled to participate in this session, was unable to atcend because of illness. Dr. Avery very kindly agreed to substitute for him.
The odontoblasts develop collagen necessary for the production of the dentin and are the first cells of the pulp to be affected by external stimuli since their processes exist in the surrounding dentin.

The larger blood vessels are centrally located in the pulp and it is of interest that they appear relatively thin walled when compared with vessels in other connective tissue areas of the body but that they are not as thin walled as vessels in some organs. The vessels of the pulp help characterize this organ as one different from any other in the body. The larger vessels have several layers of smooth muscle cells separated by a 300 to $500 \mathrm{~A}$ basal lamina which surround the endothelial cells lining these vessels. These vessels have numerous adjacent nerve fibers, many of which are myelinated. The large myelinated nerves probably function in fast conduction of about 60 to 120 meters per second, and smaller nonmyelinated nerves also can be seen near the vessels which may be sympathetic in nature and related to the dilation and constriction of these vessels.

The larger vessels and nerves branch to the peripheral pulp where capillaries and precapillaries are seen in the odontogenic regions (Figs 1, 2). The capillaries are found to have an incomplete smooth muscle coat surrounding the endothelial cells and occasionally pericapillary cells or fibroblasts are found on the surface of these small vessels. Near the predentin are thin-walled capillaries (that Dr. Frank ${ }^{1}$ has described), which have thin sections and thicker sections where pericytes or capillary-associated fibroblasts are found (Fig 1). The vessel wall near the predentin illustrates pores or fenestrations. These pores are characterized by 300 to $500 \mathrm{~A}$ openings in the cell wall that are bridged by a thin membrane which 


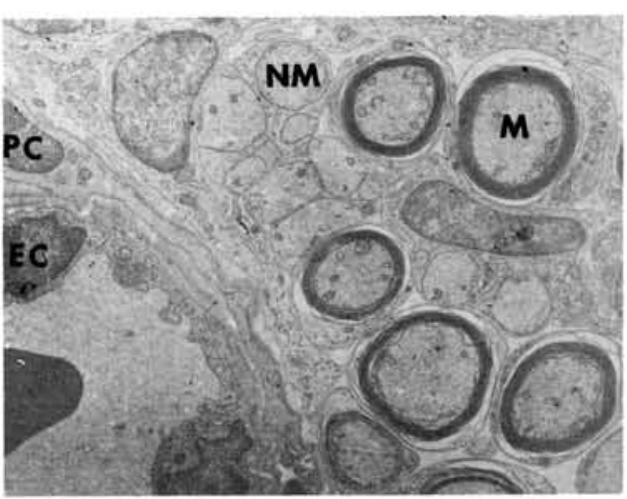

FIG 1.-Capillary of pulp surrounded by pericytes $(P C)$. Endothelial cells $(E C)$ line lumen. Nonmyelinated $(N M)$ and myelinated $(M)$ nerve fibers lie adjacent on right.

appears to be continuous on either side of the pore with the endothelial cell wall 2 (Fig 3). The fragile nature of these vessels near the predentin makes them especially vulnerable to trauma of the adjacent dentinal tissue. We are aware that the capillaries in this region are torturous and entwine about the odontoblasts, especially in the early stages of tooth formation. ${ }^{2}$ Later, after the period of rapid dentin formation, the peripheral capillaries recede into a subodontoblastic location.

The odontoblast is of interest to all of us, insofar as it is a cell that maintains the dentin vitality while functioning in dentin matrix formation throughout life. At the same time, the process of this cell becomes

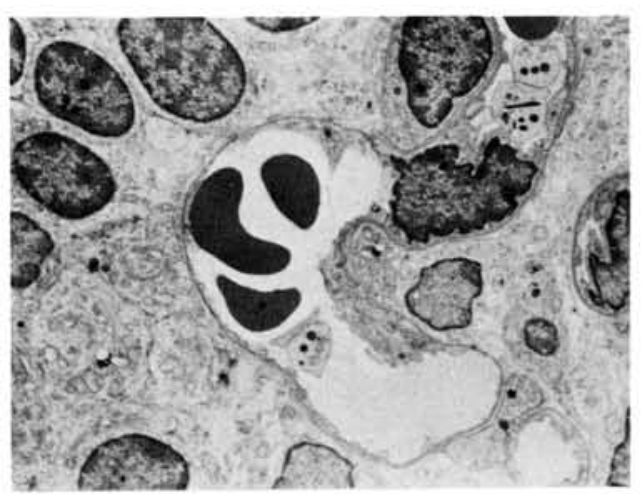

Fig 2.-Thin-walled capillary located among odontoblasts which are seen right and left. Tortuous nature of vessels in region is apparent.

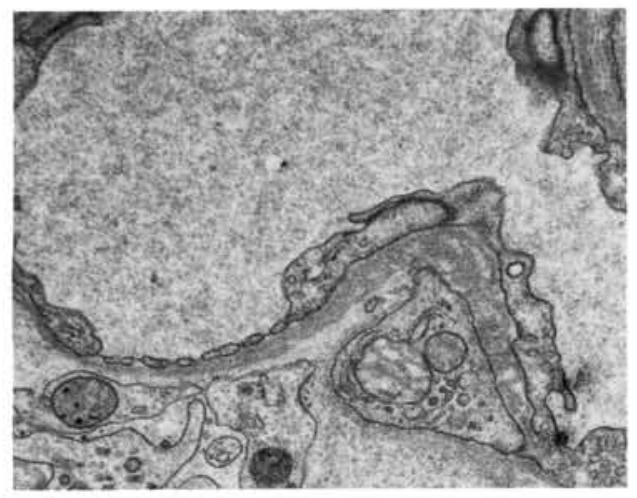

FIG 3.-Pores or fenestrations in pulpal capillary located adjacent to predentin. These 300 to $500 \mathrm{~A}$ openings are bridged by thin membrane that is continuous with endothelial cell wall.

increasingly longer until the total process is many times the size of the cell body. The cytoplasm of the odontoblast process appears nearly free of organelles and contains only occasional microvesicles, microtubules, or dark granular bodies. The cytoplasm of the processes contrast with nerve fibers that may lie adjacent to them (Fig 4). The processes are best described as containing numerous long, fine, longitudinally arranged filaments (Fig 5). The odontoblast processes have numerous branches that appear at right angles to the main processes and exist in small canaliculi extending from the dentinal tubules toward adjacent dentinal tubules (Fig 5). Thus dentin, like pulp, is a highly vital tissue capable of response to any

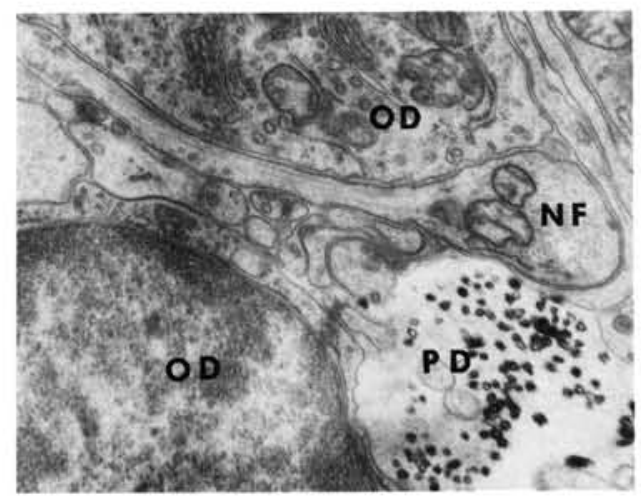

FIG 4.-Longitudinal section of nonmyelinated nerve fiber $(N F)$ between two odontoblasts $(O D)$. No Schwann cell covering appears. Predentin $(P D)$ is on right. 


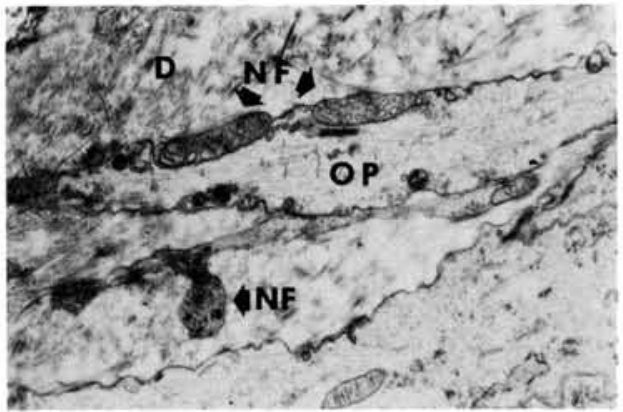

Fig 5--Nonmyelinated nerve fibers $(N F)$ are located intratubularly accompanying odonto. blastic process $(O P)$ in dentin $(D)$. Below is nerve $(N F)$ which may be lateral branching in canaliculi.

pharmacologic agent brought in contact with it.

Drs. Corpron, Cox, Lee, and $\mathrm{I}^{3}$ have reported two types of nerve endings in the area of the predentin and have observed an intimate relationship between the odontoblast and its process with the terminal portions of these intrapulpal and intratubular nerves. Nonmyelinated nerves may pass between the odontoblasts to terminate in the tubule (Fig 4). They may lie adjacent to the odontoblast process and may exhibit alternating constrictions and dilations of the nerves and the process (Fig 5). This creates an increased surface area between these two structures. This has been commented on earlier by Frank. 4 Some of these nerve endings found in this location have vesicles that are small and comparatively

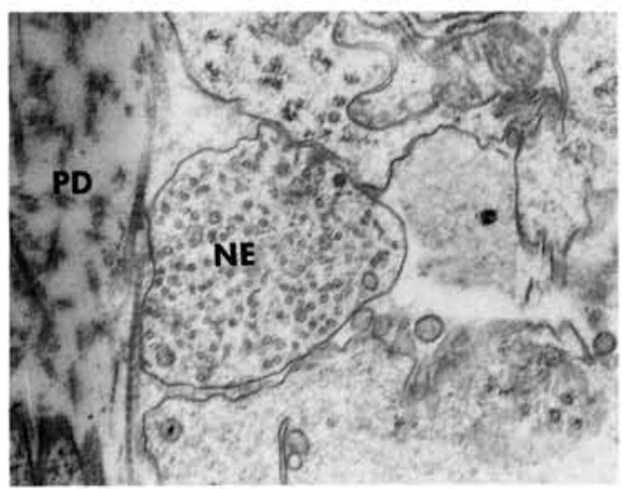

Fig 6.-Possible nerve ending $(N E)$ in pre dentin, containing numerous electron dense small vesicles.

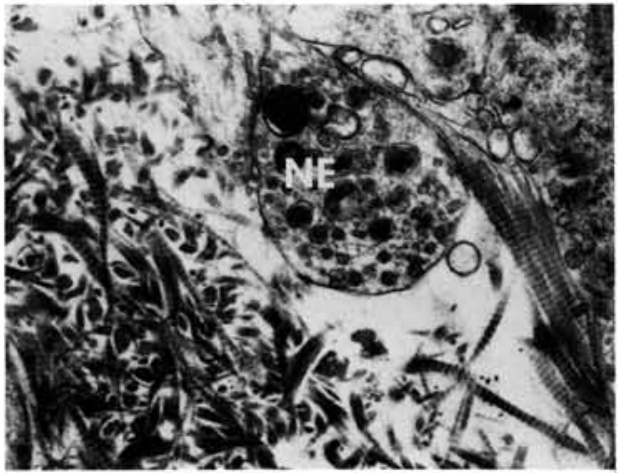

Fig 7.-Possible nerve ending $(N E)$ in predentin containing large vesicles that are extremely electron dense.

light staining, whereas others appear to have large vesicles with electron-dense granules (Figs 6, 7).

Hand $^{5}$ described ultrastructural differences in adrenergic and cholinergic nerve terminals based on the size and electron density of the granules. When an experimental animal has had 5-OH-dopamine $(33 \mathrm{mg} / \mathrm{gm}$ of body weight) injected intraperitoneally, this false neurotransmitter localizes in the adrenergic nerve endings. This substance is then precipitated by glutaraldehyde and reduced by osmic acid fixation and appears as electron-dense core vesicles of the adrenergic endings. ${ }^{6}$ These can be seen in otherwise unstained sections along the walls of these vessels.

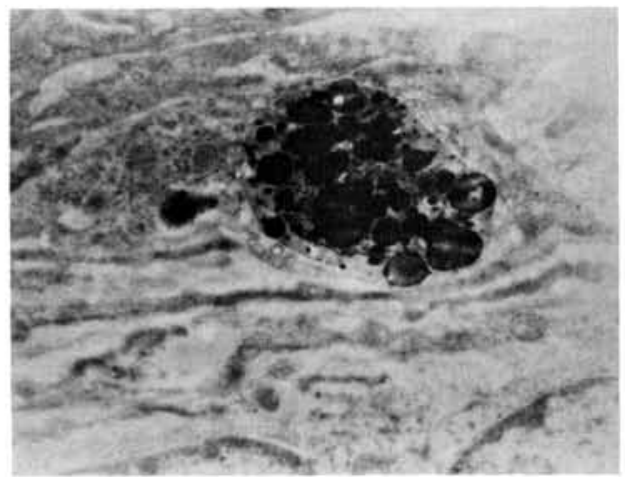

Fig 8.-Nerve ending located adjacent to blood vessel near center of pulp. Specimen was injected with 5-OH-dopamine 30 minutes before animal was killed. Section is otherwise un. stained. 


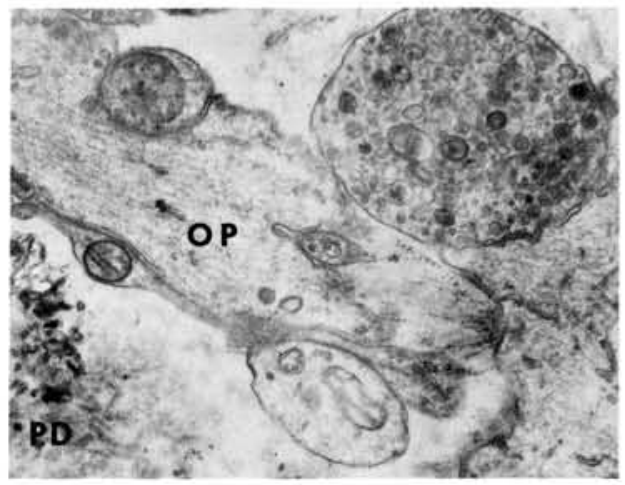

FIG 9.-Possible normal-appearing nerve ending adjacent to odontoblast process $(O P)$ and predentin $(P D) 21$ days after resection of inferior alveolar nerve.

In this case, 5-OH-dopamine had been injected 30 minutes before the animal was killed. Concentrations are found in some of the endings along the pulpal blood vessels as well as in the predentin adjacent to the odontoblast and their processes (Fig 8). It is thus concluded that some of the endings in these locations are adrenergic and not sensory in nature. To confirm the identity of nerves in the dentinal tubules, resection of the inferior alveolar nerve was done on the left side of mice, leaving the right side intact. ${ }^{7.8}$ Butcher and Taylor ${ }^{9}$ were among the first to note changes in pulps of teeth after neural resection. Degeneration of neural endings adjacent to the odontoblast processes was observed. ${ }^{8}$ In some cases, however, nerves were found still persisting among odontoblasts and in the area of the predentinal tubules even 21 days after resection (Fig 9). Most of these endings contained large vesicles of electron-dense material and thus appeared to be adrenergic in nature.

Further studies on the effect of external trauma on teeth with inferior alveolar nerve resection were carried out. Cavities of similar size and depth were placed in the right and left central incisors of rabbits 14 days after inferior alveolar nerve (IAN) resection and the pulps were evaluated 14 and 35 days later.10 Excessive amounts of dentin were found in the incisors only on the resected side. ${ }^{3}$ It is of interest that the excessive response dentin appears different in nature than the normal tubular dentin

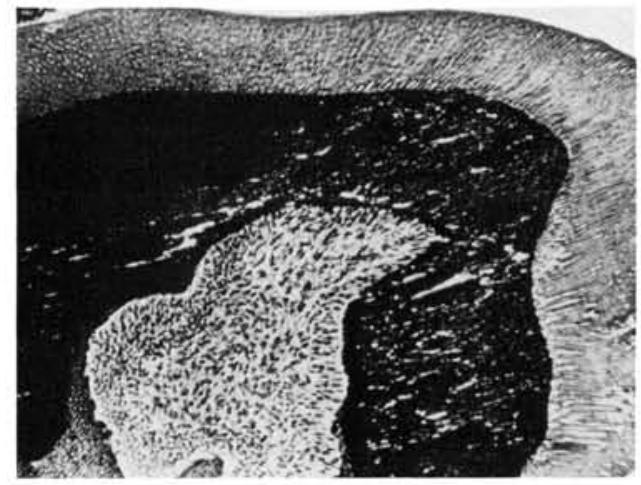

FIG 10.-Incisor cross section shows dentin formed after inferior alveolar nerve resection and amputation of tip of tooth. Dentin appears altered in structure (Silver stain).

(Fig 10). It is also of interest that the teeth lost their ability to deposit dentin only in the area underlying a cavity but formed it circumpulpally. Since the mechanism controlling formation of reparative dentin in pulps is still not clarified, it is of interest to speculate what possible effect these nerves may have on this process. Because of the finding of excessive deposition of dentin after IAN resection and stimulation, it could be theorized that a parasympathetic component traveling with the IAN also would be resected. This loss could affect the sympathetic endings located near the odontoblasts. This could be part of the system that controls reparative dentin formation. If this is true, then which are the sensory endings responsible for pain reception? Since any parasympathetic component is cholinergic it may be difficult to identify from pain receptors by use of present, commonly known techniques. It is obvious there are still many questions unanswered concerning the explanation of the control of dentin formation to external stimuli.

\section{Study of Effect of Dentin Materials on Pulp and Dentin}

The next objective is to discuss the continued need for uniform testing procedures when we conduct studies on pulpal response to restorative agents. We followed the recommended procedures as set forth by the Committee on Standard Practice of Evaluation of Dental Materials ${ }^{11}$ (Fig 11). In our 


\section{Recomended Standard Practice of Evallation} Dental Materials an Devices on Pulps

J.A.D.A. VOL. 84, FER. 1972

1. USE OF INTACT, NON-CARIOUS, NON-SIGAIFICANTLY ABRADED TEETT.

2. Class $V$ CAVITIES PREPARED AT HIGH SPEED WITH NEI No. 35 CARBIDE BURS WITI EFFICIENT WATER SPRAY COOLANT.

3. UlSE OF MATERIAL AS RECOMENDED BY MANUFACTURER.

4. ISE OF TWO CONTROLS: ZNOE. ARD SILICATE UNLINED,

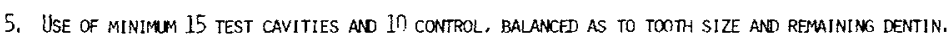

6. ONE THIRD OF TEETH EXTRACTED BETWEEN 24 - 72 HOURS FOR INITIAL RESPONSE:

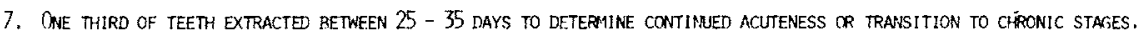

3. DNE THIRD OF TEETH EXTRACTED ABOUT EI \pm In DAYS TO DETERMINE IF LESION RESOLVED OR NOT.

9. IMMEDIATELY AFTER EXTRACTION RENOVE APICAL ONE-THIRD AND FIX WITH IT: FORMALIN. DREPARE WITH ACCEPTEI) HISTOLOGIC TECHIQUES.

current studies, intact, noncarious teeth of rhesus monkeys were used. The value of uniformly healthy teeth can be realized, although more pharmacologic agents in use today should be evaluated on the inflamed pulp as well.11 Investigators are working on a means of producing uniform inflammatory conditions for this purpose. In our attempt to attain a uniform cavity size in this study, Class $\mathrm{V}$ cavities were prepared with a No. 35 inverted cone bur using $11 / 2$ times the bur width in occluso-cervical height and depth and three times the bur width in mesiodistal diameter. We found this depth resulted in approximately $0.5 \mathrm{~mm}$ remaining dentin, and because of the ease of direct viewing, we were able to attain a fairly accurate cavity size using this method. Five test cavities for each compound were used at three time periods of three days, five weeks, and eight weeks. All compounds were placed in an equivalent number of anterior and posterior, maxillary and mandibular teeth to ensure uniform distribution in all types of teeth. New burs were used for every fourth cavity preparation with efficient water spray coolant to maintain consistent cutting conditions and minimal trauma. When each animal was killed, it was anesthetized and perfused with saline solution and phosphate-buffered Formalin (PBF). The teeth were extracted by removing the

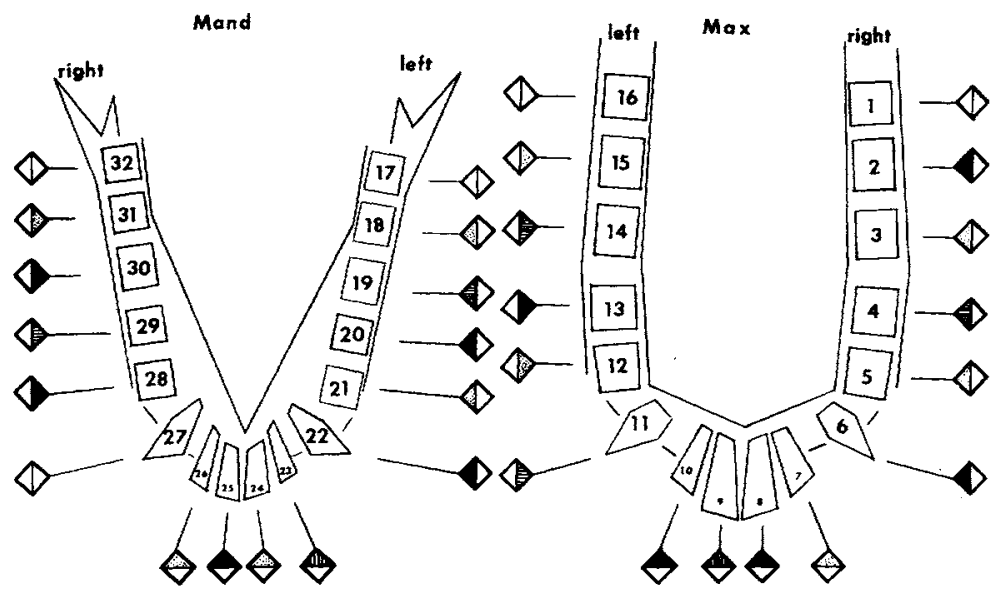

Fic 12.- Typical chart used to chart procedures carried out on rhesus monkeys. Squares indicate type of material used and whether standard depth cavity or pulp exposure was performed. 


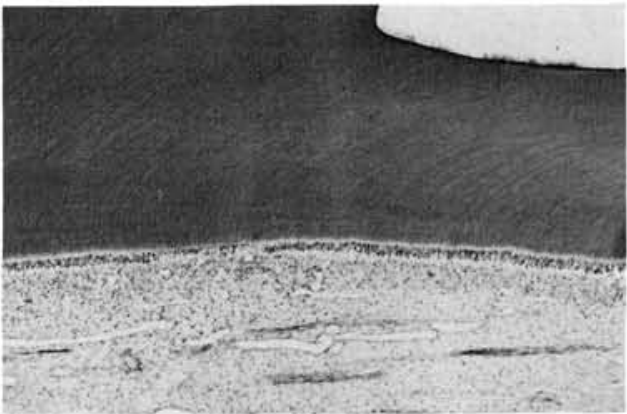

Fig 13.-Typical response to calcium hydroxide liner after three days. Disruption of odontoblasts and inflammatory cells seen in limited zone under center of cavity.

buccal plate, allowing gentle elevation of the teeth from the sockets. The apical third of the root tip was then removed with a surgical bur and the tooth placed in PBF. They were then prepared by routine histological methods using the paraffin method to allow ribboning and to facilitate serial sections through the critical areas. The restorations were placed and charted using a coded recording system for the teeth (Fig 12). The histological evaluation was done separately by two investigators. The findings of two separate operators were based on the following criteria.

A great deal of discussion could evolve about the parameters of classification of a reaction site as either slight, moderate, or severe. We have characterized a severe reaction as one with decided inflammatory cell infiltration adjacent to the cavity site; hy.

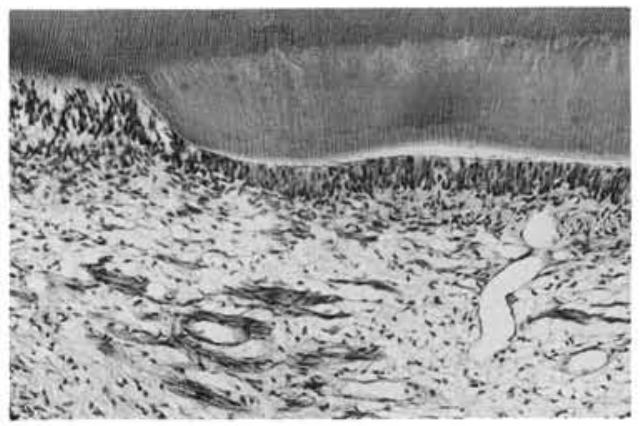

FIG 14.-Typical response to calcium hydroxide liner after five weeks. Reparative dentin is seen underlying cavity. Remaining inflammatory response was slight.

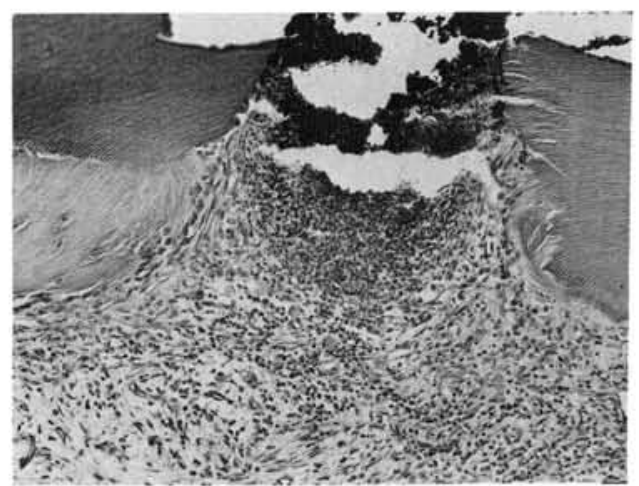

FIG 15.-Response of pulp eight weeks after placement of calcium hydroxide compound on pulp exposure. Inflammation is localized but still present. Note reparative dentin underlying floor of cavity on left but none underlying exposure.

peremia and localized abscess may be observed; hemorrhage may be seen and in this regard perfusion may enhance visualizing extravascular location of blood cells, if it is done carefully; the odontoblastic layer would be destroyed or greatly disrupted; and the predentin may be reduced or absent. A moderate reaction is characterized as a definite increase in inflammatory cells, localized hyperemia, occasional hemorrhage in the odontoblastic or subodontoblastic zone; the odontoblastic layer may be discontinuous; and the predentin may be affected. A slight reaction is characterized by a slight increase in inflammatory cells, mild hyperemia, and

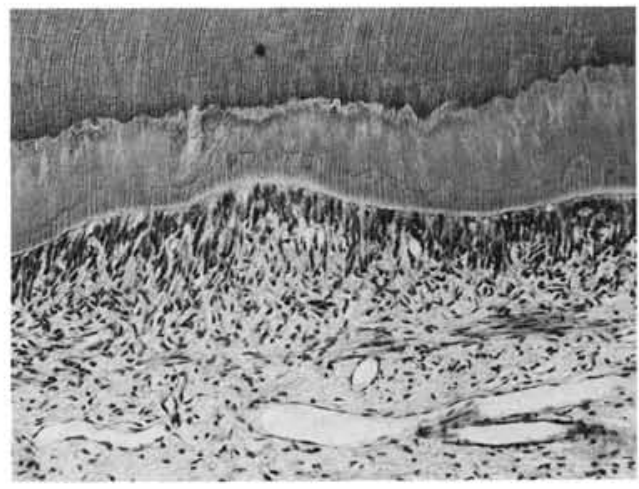

Fig 16.-Response by a composite filling five weeks after placement in standard cavity. Reparative dentin and some inflammatory cells are present. 


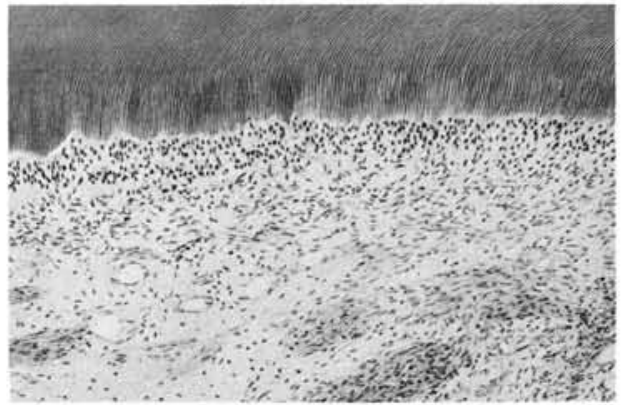

FIG 17.-Typical response to zinc oxide eugenol compound five weeks after placement in standard cavity. Only slight inflammatory response and minimal reparative dentin are pres. ent.

possibly small hemorrhages in the odontogenic zone; irregularities in the odontoblastic layer may be observed. Today, most investigators evaluating dental materials are using the guidelines of the recommended Standard Practices, which will allow evaluation of results with greater ease and accuracy. With the foregoing criteria well in mind in our studies, at least two investigators would examine each slide separately and later final analysis would be compared. Where disagreement arose, a third investigator would evaluate and there would be a reevaluation by all three investigators until agreement was reached. Both remaining and reparative dentin was measured with an optical micrometer.

The comparative response of the pulp to various restorative materials in the current study is shown in Figures 13 and 14. All

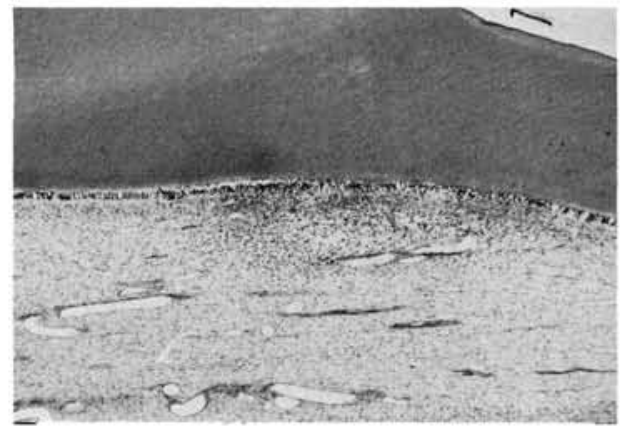

FIG 18.-Typical response to silicate three days after placement in standard depth cavity. Localized inflammatory response and disruption of odontoblastic cell layer.

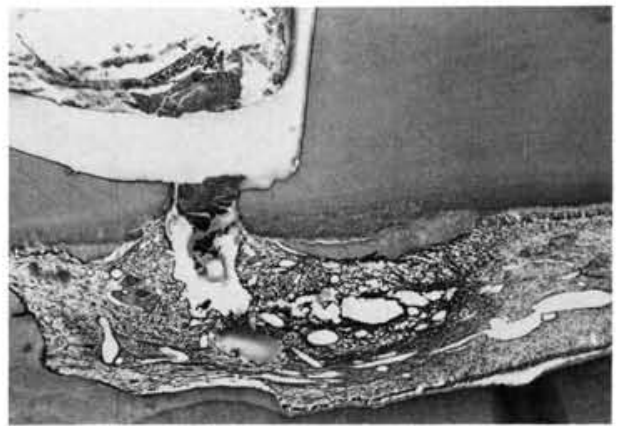

FIG 19.-Response seen eight weeks after placement of silicate on exposure of pulp. Although response is severe, some reparative dentin formed under floor of cavity to left and right of exposure.

compounds cause an initial response but this may be slight by the time of the five-week analysis period as in the case of zinc oxide eugenol or polycarboxylates; it may be mod. erate for composites or calcium hydroxide compounds or severe as in the case of silicates. Each group of compounds has a number of interesting characteristics. This is particularly true of the calcium hydroxide liners. Most calcium hydroxide compounds cause a moderate initial reaction (Fig 13); at later intervals the reaction decreases and a layer of reparative dentin is seen (Fig 14). The amount of reparative dentin deposited is greater with some calcium hydroxide compounds than others. The reaction of the exposed pulp to calcium hydroxide produces inflammation of longer duration and severity than in the nonexposed. We, of course, are

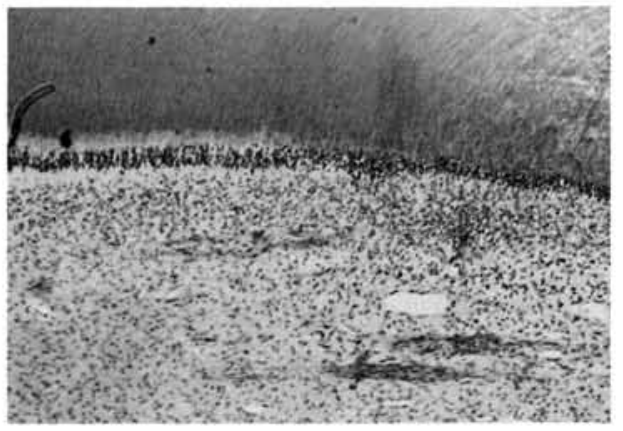

FIG 20.-Typical appearance of pulp under. lying cavity in which polycarboxylate was placed. Inflammatory cells appear in area although odontoblasts are not disrupted. 
considering the normal pulp in these studies. It has been shown that calcium hydroxide may not have any beneficial effects on healing on the exposed inflamed pulp. ${ }^{12}$ Some calcium hydroxide compounds do not induce reparative dentin formation on exposures as readily as others (Fig 15). This has been investigated by Stanley and Lundy. ${ }^{13}$ It is of interest that most calcium hydroxide compounds will induce reparative dentin underlying a cavity much more readily than in an exposure (Fig 15) .

After five weeks, most modern day composites will induce reparative dentin formation and may show signs of slight inflammatory conditions (Fig 16). In comparison, zinc oxide shows little reparative dentin formation but there is a persistence of inflammatory cells underlying the cavity floor (Fig 17). The initial response of a normal pulp to a silicate restoration is usually mild in a cavity with $0.5 \mathrm{~mm}$ remaining dentin (Fig 18). The closer to the pulp, the greater is the reaction, however. There is more variation in the response that different composites cause when they are placed in contact with the pulp rather than in a cavity. Some may cause as severe a reaction as silicate (Fig 19). The quality and amount of reparative dentin should also be considered in pulpal evaluation. On an exposed pulp, silicate causes a severe reaction with abscess formation (Fig 19). Silicate will stimulate dentin formation underlying a cavity (Fig 19). It is of interest that silicates, composites, and calcium hydroxide compounds all form quite regular reparative dentin underlying intact dentin but only some calcium hydroxide compounds cause reparative dentin formation when these chemical substances are placed in contact with the pulp. Polycarboxylate cement was introduced by Smith in $1968^{12}$ and its biologic properties have not yet been extensively investigated.14-16 Our observations showed a slight reaction at three days (Fig 20) and the same at five and eight weeks. Reparative dentin did appear at the later time periods. These compounds thus responded similarly to zinc oxide eugenol cements except for the addition of reparative dentin in the case of the polycarboxylate compound. When these compounds were placed in contact with the pulp, the inflam-

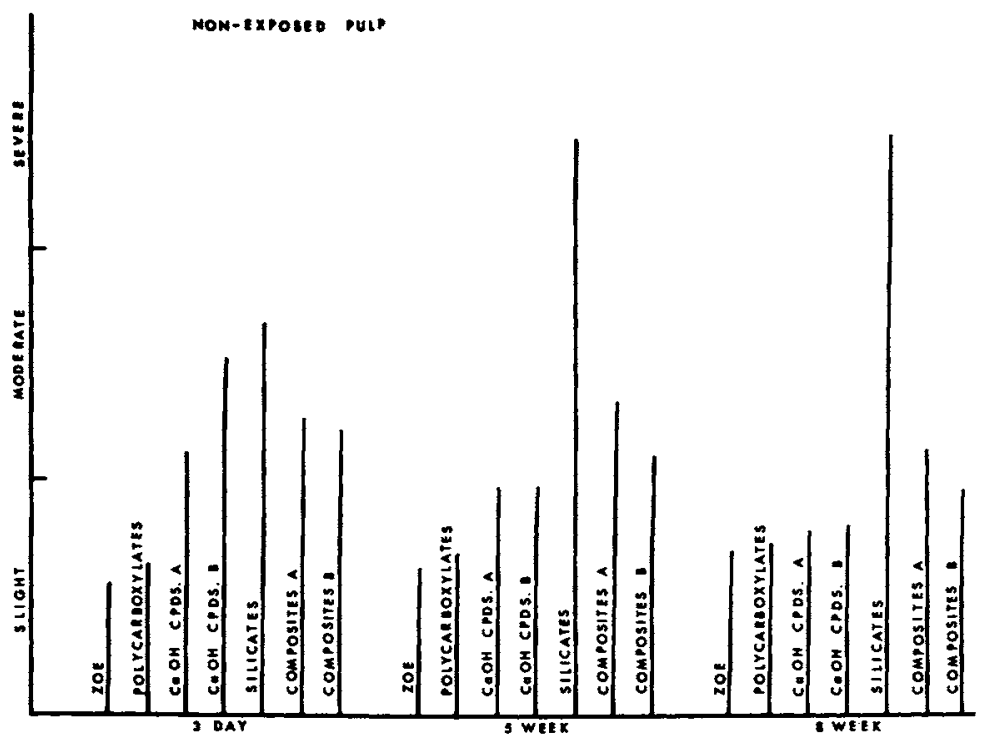

Fic 21.-Graph indicates response of pulp of rhesus monkeys when dental materials were placed in standard depth cavities. Calcium hydroxide and composites were indicated as compounds $A$ and $B$ to denote different compounds. 


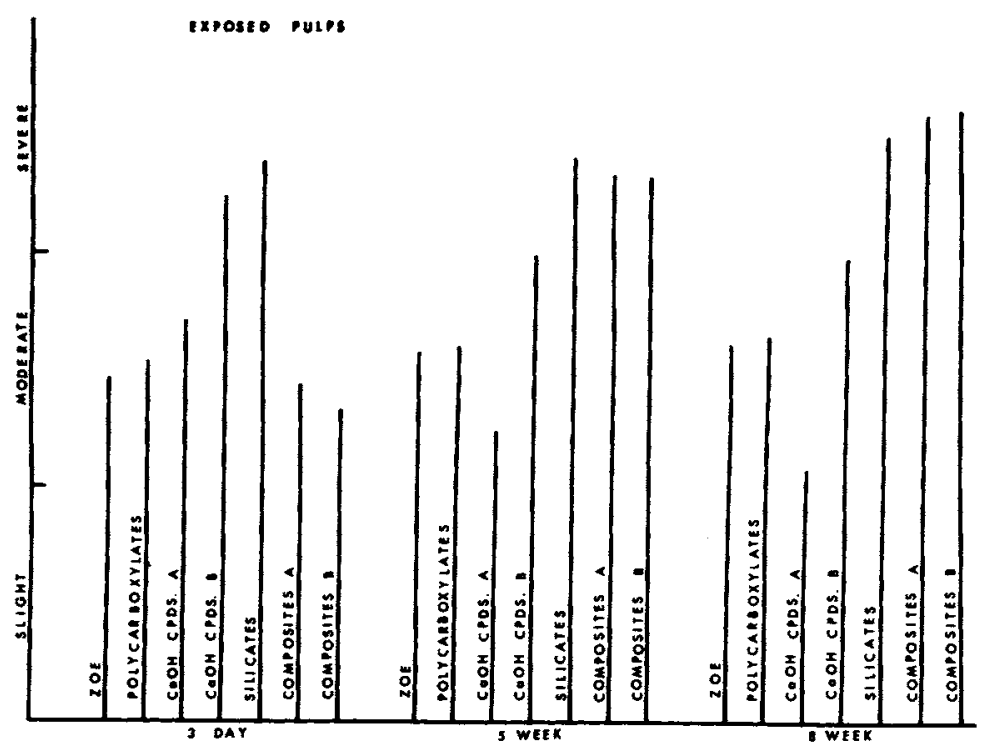

Fig 22.-Response of pulp of rhesus monkeys when dental materials were placed in contact with pulp. Increase in severity of response is noted with most compounds.

matory process increased over cavity placement but was moderate at the five- and eight-week time periods. The response in both the nonexposed and exposed situations was similar to that of zinc oxide eugenol.

In summary, the most important point that I hope I have made is to encourage a standard reproducible method of testing pharmacologic agents on the dental pulp. The guidelines have been published, and they present a model which we used and improved on as additional information became available. Classification of results is another area of concern, as some use a numerical score and others use descriptive terms. Again, a definition of terms will help clarify the system for the reader. The final two charts are presented to illustrate in summary form the results attained in the present study of agents placed in cavities and in exposed pulps (Figs 21, 22). The several calcium hydroxide and composite compounds are separated into two response categories to illustrate the differences some of these compounds elicit. In our experience they seem to fall into two groups. The more recent compounds reaching the market appear to be formulated with care concerning their material as well as biologic properties. This is a tribute to the many investigators at this conference and others who are striving to present the dental profession with the best possible restorative materials.

The author thanks his associates: Drs. Richard Corpron, Charles Cox, and S. D. Lee concerning the ultrastructural studies and Drs. Ronald and Donald Heys and Wendell Racette on the pulp testing study.

\section{References}

1. Frank, R.M.: Reactions of Dentin and Pulp to Drugs and Restorative Materials, $J$ Dent Res 54 (Special Issue B) : 176-187, 1975.

2. Corpron, R.E.; AVery, J.K.; and LEe, S.D.: Ultrastructure of Terminal Pulpal Blood Vessels in Mouse Molars, Anat Rec 179: 527-542, 1974.

3. Avery, J.K.; CoX, C.F.; CorPron, R.E.; and LEE, S.D.: Ultrastructural Observations of Neural Endings in Mouse Molar Pulps, $J$ Dent Res 52 (Special Issue) : Abstract No. 106, 1973.

4. Frank, R.: The Ultrasiructural Relationship Between the Odontoblast, Its Process and the Nerve Fibers, in Symons, N.B.B. (ed): Dentin and Pulp, Their Structure and Relations, London: $\mathbf{E}$ \& $S$ Livingstone Ltd., 1968, pp 115-145.

5. HaNd, A.R.: Adrenergic and Cholinergic Nerve Terminals in the Rat Parotid Gland. Electron Microscopic Observations on Per- 
manganate-fixed Glands, Anat Rec 173: 131$140,1972$.

6. Avery, J.K.; Cox, C.F.; Corpron, R.E.; and Trefz, B.R.: Distribution of Adrenergic and Cholinergic Nerve Endings in Mouse Molars Utilizing Morphological and Cytochemical Analysis, J Dent Res 52 (Special Issue) : Abstract No. 75, 1974.

7. Avery, J.K.; Corpron, R.E.; LeE, S.D.; Cox, C.F.; and YU, H.: Ultrastructural Variations of Intradental Nerves Following Long Term Mandibular Nerve Resection, abstracted, IADR Program and Abstracts of Papers, No. 205, 1972.

8. Corpron, R.E.; Avery, J.K.; and Cox, C.F.: Ultrastructure of Intradental Nerves after Resection of the Inferior Alveolar Nerve in Mice, J Dent Res 51: 673, 1972.

9. Butcher, E.O., and TAylor, A.C.: The Effects of Denervation and Ischemia on the Teeth of the Monkey, J Dent Res 30: 265275,1951 .

10. AverY, J.K.; Cox, C.F.; and Corpron, R.E.: The Effects of Nerve Resection and Cavity
Preparation and Restoration on Response Dentin Formation in Rabbit Incisors, $\mathrm{Arch}$ Oral Biol, in press.

11. Myers, G.E.: Council on Dental Materials and Devices. Recommended Standard Practice of Evaluation of Dental Materials and Devices on Pulps, JADA 84: 388-390, 1972.

12. Tronstad, L., and MJör, I.A.: Capping of the Inflamed Pulp, Oral Surg 34: 477-485, 1972.

13. Stanley, H.R., and Lundy, T.: Dycal Therapy for Pulp Exposures, Oral Surg 34: 818-827, 1972.

14. SAFER, D.S.; Avery, J.K.; and Cox, C.F.: Histopathologic Evaluations of the Effects of New Polycarboxylate Cements on Monkey Pulps, Oral Surg 33: 966-975, 1972.

15. Jendresen, M.D., and Trowbridge, H.O.: Biologic and Physical Properties of a Zinc Polycarboxylate Cement, I Prosthet Dent 28: 264-271, 1972.

16. El-KAFRAWY, A.H.; DickeY, D.M.; Mitchell, D.F.; and PhILliPs, R.W.: Pulp Reaction to a Polycarboxylate Cement in Monkeys, $J$ Dent Res 53: 15-19, 1974. 